\title{
Test results on hybrid photodiodes
}

\author{
N. Kanaya ${ }^{\mathrm{a}, *}$, Y. Fujii ${ }^{\mathrm{e}}$, K. Hara ${ }^{\mathrm{d}}$, T. Ishizaki ${ }^{\mathrm{d}}$, F. Kajino ${ }^{\mathrm{b}}$, K. Kawagoe ${ }^{\mathrm{a}}$,

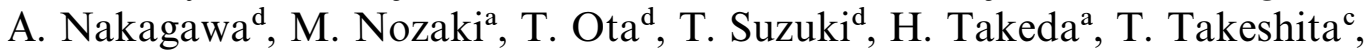 \\ T. Toeda ${ }^{c}$, Y. Yamada ${ }^{\mathrm{b}}$, C. Yokoyama ${ }^{\mathrm{a}, 1}$ \\ a Department of Physics, Kobe University, Kobe, Hyogo 657-8501, Japan \\ ${ }^{\mathrm{b}}$ Department of Physics, Konan University, Kobe, Hyogo 658-8501, Japan \\ ${ }^{\mathrm{c}}$ Department of Physics, Shinshu University, Matsumoto, Nagano 390-8621, Japan \\ ${ }^{\mathrm{d}}$ Institute of Physics, Tsukuba University, Tsukuba, Ibaraki 305-8571, Japan \\ e Institute of Particle and Nuclear Studies, High Energy Accelerator Research Organization (KEK), Tsukuba, Ibaraki 305-0801, Japan
}

Received 28 April 1998; received in revised form 14 September 1998

\begin{abstract}
We have made various measurements on four different types of Hybrid PhotoDiodes (HPDs) with and without magnetic fields. We confirmed that all the four HPDs were operational with a magnetic field with a strength of at least $1.5 \mathrm{~T}$ if the device axis was adjusted approximately parallel to the magnetic field direction. Origins of the gain variation in magnetic fields were also investigated. (C) 1999 Elsevier Science B.V. All rights reserved.
\end{abstract}

PACS: $85.60 . \mathrm{Gz} ; 85.60 . \mathrm{Ha} ; 85.60 . \mathrm{Dw}$

Keywords: HPD; Photon detector; Calorimetry

\section{Introduction}

Photon detection devices which are operational in strong magnetic fields with high sensitivity are in demand in many application fields, especially in high energy physics. For example, the photon detection device for the calorimetry, which will be located in a strong magnetic field as designed for some future collider experiments, should have such

\footnotetext{
* Corresponding author. Tel.: + 8178803 0543; fax: + 8178 803 0724; e-mail: naoko@phys.kobe-u.ac.jp.

${ }^{1}$ Present address. Norikura Observatory, Institute for Cosmic Ray Research, University of Tokyo, Japan.
}

performance. The Hybrid PhotoDiode (HPD) is one of the promising candidates for such applications. It comprises a photocathode and a PIN (or avalanche) silicon diode in a vacuum tube. A high voltage is applied between the photocathode and the diode so that photoelectrons from the photocathode are electrostatically accelerated towards the diode to achieve a higher gain.

The advantages of the device include:

1. the HPD can be used in strong magnetic fields without losing its gain significantly, if the device axis is set approximately parallel to the magnetic field direction, 
2. the HPD potentially has a good single photoelectron response, as a consequence of the highgain single-step amplification process,

3. the time response of the HPD is as fast as those of usual photomultipliers, and

4. the HPD is compact in length compared with usual photomultipliers because it essentially consists of only two components; a photocathode and a diode.

Several groups have reported R\&D studies on HPDs and their applications in recent years [1-12]. In this paper we report test results on the performance of four different types of HPDs with emphasis on their behavior in magnetic fields. Table 1 summarizes basic parameters of the HPDs given in their data sheets.

PP0350 and PP0380 developed by DEP (Delft Electronic Products ${ }^{2}$ ) are proximity focused HPDs with a PIN diode of a large effective area and a small gap between the photocathode and the diode. They should be thus operational in strong magnetic fields. PP0350 is of a high gain type, for which the photocathode voltage (the high voltage between the photocathode and the diode) can be applied up to $15 \mathrm{kV}$. PP0380 is a multi-pixel HPD, where the readout pad of the PIN diode is divided into seven pixels; an inner pixel of $56 \mathrm{~mm}^{2}$ and six outer pixels of $72 \mathrm{~mm}^{2}$ each. The signals of the pixels can be individually read out. R7100U and R7110U [12] are very compact HPDs developed by Hamamatsu (Hamamatsu Photonics K.K. ${ }^{3}$ ), with their dimensions being only $20 \mathrm{~mm}$ in diameter and $17 \mathrm{~mm}$ in length. R7100U uses a PIN diode, while R7110U uses an avalanche photodiode (APD) and thus has a larger gain. They are electrostatic focusing HPDs: the electric field is focused so that photoelectrons from the photocathode are efficiently collected to the diode, whose effective area is smaller than that of the photocathode. Therefore, the Hamamatsu HPDs are more sensitive to magnetic fields than the proximity focused HPDs.

\footnotetext{
${ }^{2}$ Delft Electronische Producten, Dwazziewegen 2, Roden, Postbus 60, 9300 AB Roden the Netherlands.

${ }^{3}$ Hamamatsu Photonics K.K., 314-5 Shimokanzo, Toyookavillage, Iwata-gun, Shizuoka 438-0126 Japan.
}

In the following, we firstly report results on the general performance of the HPDs without magnetic fields, and then report the measurements in magnetic fields.

\section{Tests without magnetic field}

\subsection{Setup}

Fig. 1 schematically shows a setup for the measurements without magnetic field. The light source was a blue LED $^{4}$ with a peak emission wavelength of $470 \mathrm{~nm}$. The light spot size at the photocathode was determined by the diameter of an aperture inserted between the LED and the photocathode. The number of photons was controlled by the driving pulse of the LED. The output signal was directly fed into a charge-sensitive CAMAC ADC.

Another setup with amplifiers in signal readout (Fig. 2) was used when an HPD was operated with a low gain or when a low-intensity light source was used. The LED light illuminated the HPD photocathode through an optical fiber of $200 \mu \mathrm{m}$ in diameter and $10 \mathrm{~m}$ in length. This was to prevent the amplifiers from picking up the noise from the driving circuit of the LED. A peak-hold CAMAC ADC (PHADC) was used to digitize the amplified signal.

\subsection{Gain and applied voltages}

Photoelectrons emitted from the photocathode are accelerated by the electric field and bombard the diode. The photoelectrons lose part of their energies in passing through the surface dead region before reaching the depletion region of the diode. The HPD gain depends on the kinetic energy of photoelectrons when they enter the depletion region. Therefore, the gain $G$ of an HPD with a PIN diode is related to the photocathode voltage $V_{\mathrm{c}}$ in the following simple formula when $V_{\mathrm{c}}$ is sufficiently larger than $V_{\text {th }}$.

$$
G=e\left(V_{\mathrm{c}}-V_{\mathrm{th}}\right) / E_{\text {pair }},
$$

\footnotetext{
${ }^{4}$ NSPB 520S, manufactured by Nichia Chemical Industries Ltd., 491 Kaminaka-cho, Anan-shi, Tokushima 774-0044 Japan.
} 
Table 1

Basic parameters of the HPDs

\begin{tabular}{|c|c|c|c|c|}
\hline Parameters & PP0350 & PP0380 & R7100U & $\mathrm{R} 7110 \mathrm{U}$ \\
\hline Diode type & PIN & PIN & PIN & APD \\
\hline Number of pixels & 1 & 7 & 1 & 1 \\
\hline Dimensions (mm) & $35 \phi \times 31$ & $50 \phi \times 21$ & $20 \phi \times 17$ & $20 \phi \times 17$ \\
\hline Photocathode diameter (mm) & 18 & 25 & 8 & 8 \\
\hline Diode diameter (mm) & 18 & 25 & 7 & 3 \\
\hline Cathode-diode gap (mm) & 14.3 & 5.0 & 12 & 12 \\
\hline Maximum $V_{\mathrm{c}}(\mathrm{kV})$ & 15 & 10 & 8 & 8 \\
\hline Maximum $V_{\mathrm{r}}(\mathrm{V})$ & 250 & 150 & 150 & 150 \\
\hline Quantum efficiency $^{\mathrm{a}}(\%)$ & 25 & 22 & 15 & 15 \\
\hline Rise time (ns) & 4 & 6 & 2.4 & 1.3 \\
\hline
\end{tabular}

${ }^{\text {a }}$ Maximum quantum efficiencies at wavelengths of 440, 400, 420 and 420 nm, respectively.

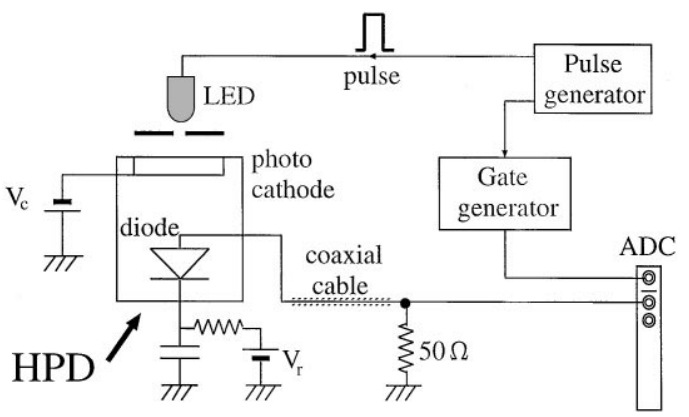

Fig. 1. Schematic view of a setup without magnetic field.

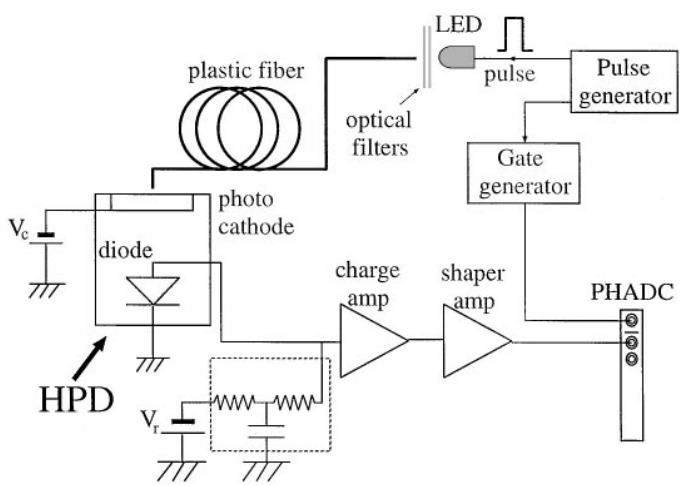

Fig. 2. Schematic view of a setup where amplifiers are used for the signal readout.

where $e$ is the electron charge, $e V_{\mathrm{th}}$ is the energy loss of the photoelectrons when they traverse the surface dead layer, and $E_{\text {pair }}(=3.6 \mathrm{eV})$ is the energy to generate an electron-hole pair in the depletion re- gion of the diode. For an HPD with an APD, the relation is modified to

$G=G_{\mathrm{a}}\left(V_{\mathrm{r}}\right) \times e\left(V_{\mathrm{c}}-V_{\mathrm{th}}\right) / E_{\text {pair }}$,

where $G_{\mathrm{a}}$ is the avalanche gain being a function of the reverse bias voltage $V_{\mathrm{r}}$. The relations of the measured gain $G$ and the photocathode voltage $V_{\mathrm{c}}$ are shown for the four HPDs in Fig. 3. In this measurement the LED light illuminated the central part of the HPD photocathode with a spot size of $2 \mathrm{~mm} \phi$. By fitting the measured gains to the above formula, the threshold voltages $V_{\text {th }}$ were determined. The absolute gains at the nominal photocathode voltages and the threshold voltages are listed in Table 2.

The average number of photoelectrons must be estimated in order to evaluate the absolute gain. For Hamamatsu HPDs this was done by using the results of photoelectron counting (see Section 2.4). The average number of photoelectrons was also estimated by the pulse height distribution for the same light source measured by a photomultiplier, and was consistent with that evaluated by the photoelectron counting. We estimated the average number for the DEP HPDs taking account of the difference of quantum efficiencies between the DEP and Hamamatsu HPDs.

Among the four HPDs, only R7110U has an APD. Fig. 4 shows the gain of R7110U where the photocathode voltage was fixed to $8 \mathrm{kV}$ and the reverse bias voltage $V_{\mathrm{r}}$ was varied. The avalanche gain factor $G_{\mathrm{a}}$ of R7110U was measured to be 33 at $V_{\mathrm{r}}=150 \mathrm{~V}$. 

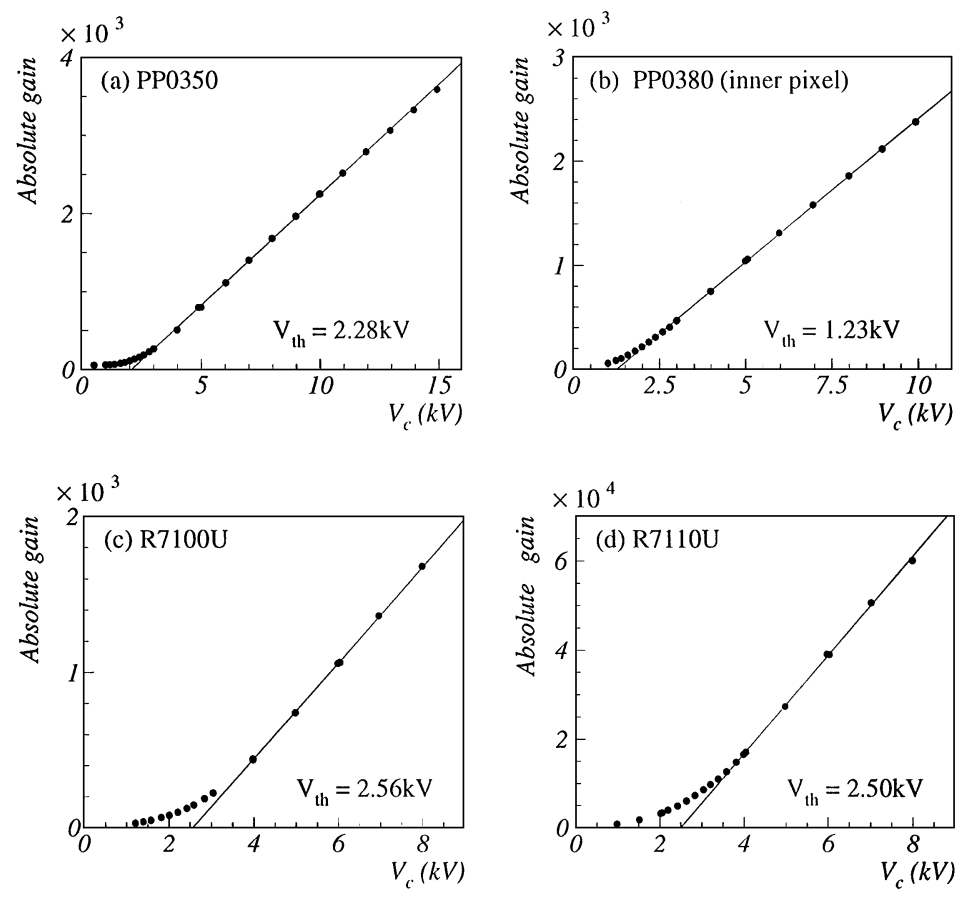

Fig. 3. Relations of the gain $G$ and the photocathode voltage $V_{c}$; (a) PP0350, (b) PP0380, (c) R7100U, and (d) R7110U, which was operated at $V_{r}=150 \mathrm{~V}$.

Table 2

The absolute gain at the nominal photocathode voltage. The reverse bias voltage of $\mathrm{R} 7110 \mathrm{U}$ was set to $150 \mathrm{~V}$. The threshold voltage $V_{\text {th }}$ is obtained from a linear fit

\begin{tabular}{llll}
\hline & Gain & Nominal $V_{\mathrm{c}}(\mathrm{kV})$ & $V_{\text {th }}(\mathrm{kV})$ \\
\hline PP0350 & $3.5 \times 10^{3}$ & 15 & 2.28 \\
PP0380 & $2.4 \times 10^{3}$ & 10 & 1.23 \\
R7100U & $1.7 \times 10^{3}$ & 8 & 2.56 \\
R7110U & $6.0 \times 10^{4}$ & 8 & 2.50 \\
\hline
\end{tabular}

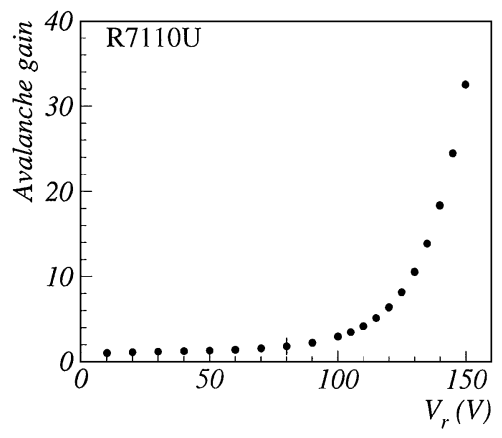

Fig. 4. The relation of the avalanche gain and the reverse bias voltage $V_{r}$ for R7110U with $V_{c}=8 \mathrm{kV}$.

diameter of the light spot was set to $1 \mathrm{~mm}$. The grid size of the position scan was 1.2 and $0.48 \mathrm{~mm}$ for the DEP and Hamamatsu HPDs, respectively. We took the data by using a charge-sensitive CAMAC ADC without amplifiers.

Fig. 5 shows the result of the position scan for PP0350 which has the largest effective area of the 

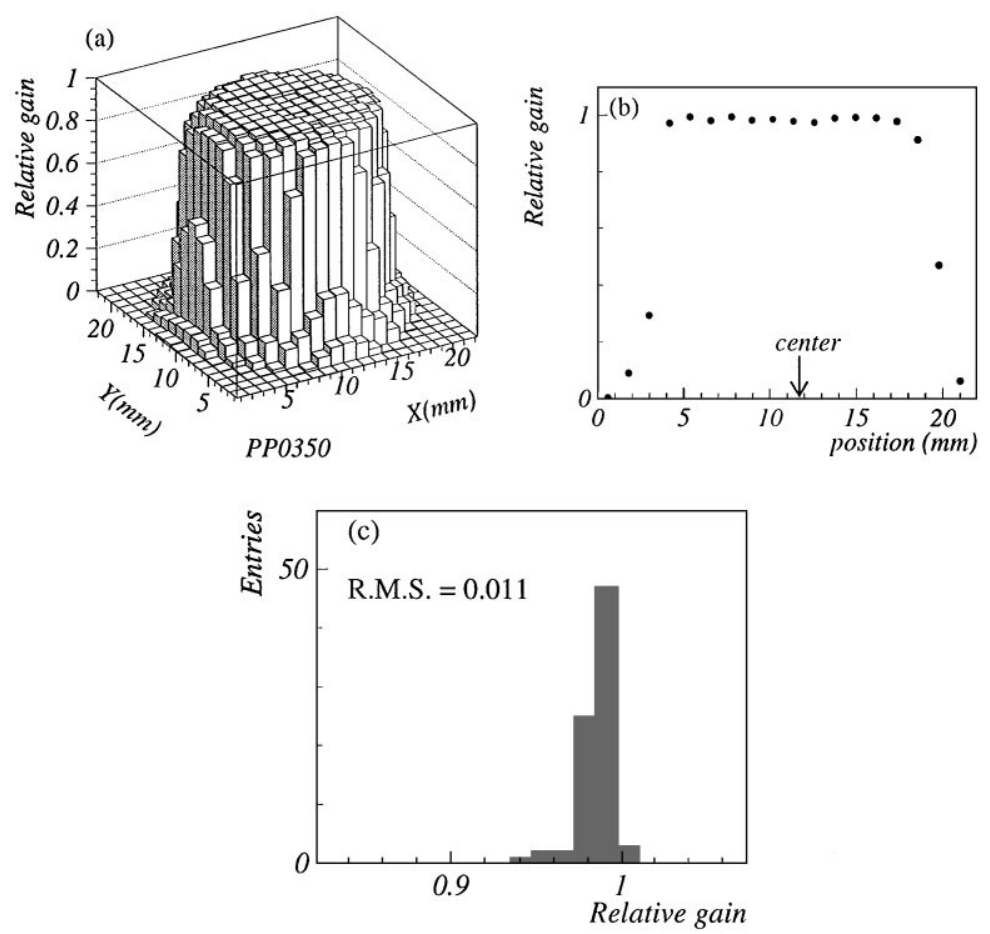

Fig. 5. Relative gains at different points of PP0350; (a) a lego plot of $x-y$ scan, (b) a scan along a center line, and (c) the distribution of PP0350 gains within a circle of $12 \mathrm{~mm} \phi$. The arrow in (b) indicates the center of the HPD.

diode. The standard deviation of the gain within a circle of $12 \mathrm{~mm} \phi$ was found to be $1.1 \%$ as shown in Fig. 5c.

Fig. 6a shows the position dependence of the sum of the seven signals of PP0380. For the line scan (Fig. 6b), the light spot size and scan pitch were changed to 0.2 and $0.12 \mathrm{~mm}$, respectively. This was to investigate the fine structure at the pad gaps of the multi-pixel HPD. Cross-talk signals were observed at the boundaries of the diode pixels. The cross-talk is assumed to be mainly caused by the photoelectrons backscattered at the surface of the diode $[13,14]$.

Figs. 7 and 8 show the results of the Hamamatsu HPDs. The standard deviation of the gain within a circle of $4 \mathrm{~mm} \phi$ was measured to be $2.2 \%$ and $3.3 \%$ for R7100U and R7110, respectively.

\subsection{Photoelectron counting}

A low-intensity light source was used to test the feasibility of photoelectron counting with the
Hamamatsu HPDs. For this purpose, we inserted a thick optical filter between the blue LED and the optical fiber. For the signal readout we used charge amplifiers; a combination of a preamplifier DEGITEX HIC1560 and a pulse shaping amp EG\&G 579 for R7100U, and a preamplifier EG\&G 142A for R7110U. Fig. $9 \mathrm{a}$ and $\mathrm{b}$ shows the pulse height distributions of R7100U and R7110U, respectively. Several photoelectron peaks are clearly seen in both the histograms. The peaks of R7110U are narrower than those of R7100U, as R7110U has a better signal-to-noise ratio owing to the avalanche gain.

\section{Tests in magnetic fields}

We performed tests of the HPDs in magnetic fields to check their response in strong magnetic fields, and to obtain better understanding of their operation mechanism in such conditions. The gain reduction may roughly be understood by the 

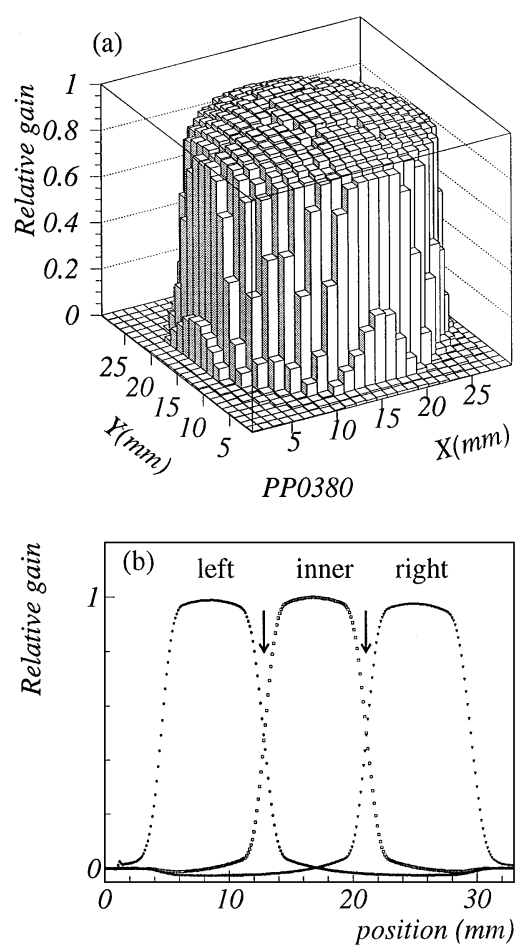

Fig. 6. Relative gains at different points of PP0380: (a) a lego plot of $x-y$ scan and (b) a scan along a center line where the signals of the three pixels (left, inner, and right) are plotted separately. The arrows in (b) indicate the boundary positions of the pixels.

cycloid motion of photoelectrons in the electric and magnetic fields. However, in order to fully understand the behavior of the HPDs, other effects such as energy loss of photoelectrons at the surface dead layer of the diode and backscattering of photoelectrons at the photocathode surface have to be taken into account.

\subsection{Setup}

We tested the HPDs using a normal conducting magnet at KEK, High Energy Accelerator Research Organization. The maximum magnetic field strength was $1.6 \mathrm{~T}$. The setup is shown schematically in Fig. 10. The blue LED was used as the light source. The variation of the light intensity was found to be less than $0.8 \%$ for any LED tilt angle and any magnetic field strength. The surface of the

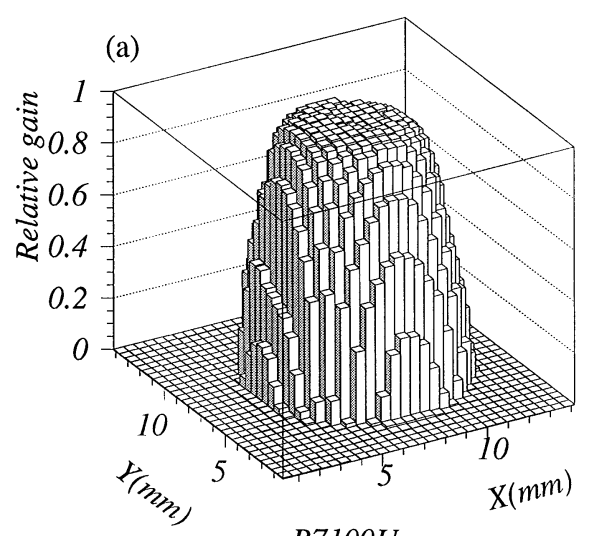

$R 7100 U$

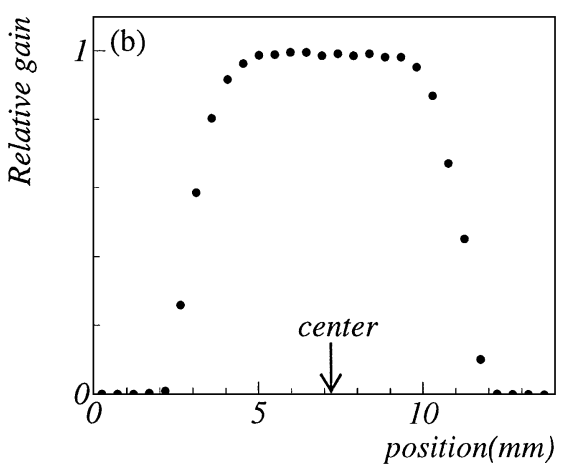

Fig. 7. Relative gains at different points of R7100U: (a) a lego plot of $x-y$ scan and (b) a scan along a center line. The arrow in (b) indicates the center of the HPD.

HPD photocathode was masked so that only the central part served as a light window. The light source and one of the HPDs were mounted in a shield box together with a light diffuser between them. The box was located in the magnet gap, and was able to be rotated to change the tilt angle $\theta$, the angle between the HPD axis and the magnetic field direction.

\subsection{Normal operation mode}

We measured the HPD gains in magnetic fields with different strengths and tilt angles. The HPDs were operated with their nominal voltages.

Fig. 11a-d shows the results. Some of the numerical values are listed in Tables 3 and 4. As are shown in the figures and tables, all the four HPDs are confirmed to be operational in strong magnetic 



Fig. 8. Relative gains at different points of R7100U: (a) a lego plot of $x-y$ scan and (b) a scan along a center line. The arrow in (b) indicates the center of the HPD.

fields, at least up to $B=1.5 \mathrm{~T}$, if they are used in certain $\theta$ ranges. As expected, the Hamamatsu HPDs are more sensitive to the magnetic fields.

Fig. 12 shows the gain as a function of the photocathode voltage for PP0380 at $\theta=0^{\circ}$. It was found that the general relation between the gain and the photocathode voltage was kept in the magnetic field, although the slope was modified.

\subsection{Photodiode mode and phototube mode}

To further investigate the effect of the magnetic field, the HPDs were operated in two special modes, namely photodiode mode and phototube mode.

In the photodiode mode an HPD was operated as a photodiode, where the reverse bias voltage was applied and the photocathode voltage was set to
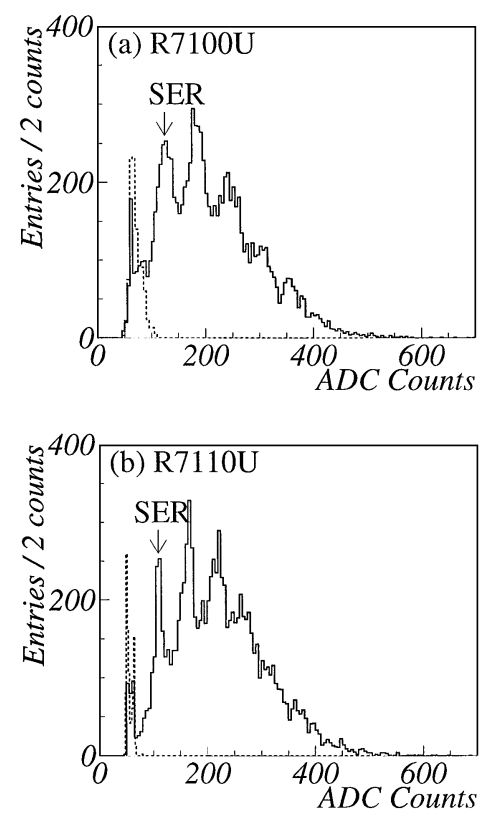

Fig. 9. The response of (a) R7100U and (b) R7110U to a low intensity light source (solid histograms). The dashed histograms are the distributions without the light source. Single photoelectron peaks are indicated by SER (Single Electron Response).



Fig. 10. Schematic view of the setup used for the tests in magnetic fields.

zero. Photoelectrons emitted from the photocathode could not reach the depletion region of the diode because of the surface dead region, and the signal pulse was generated only by photons which 

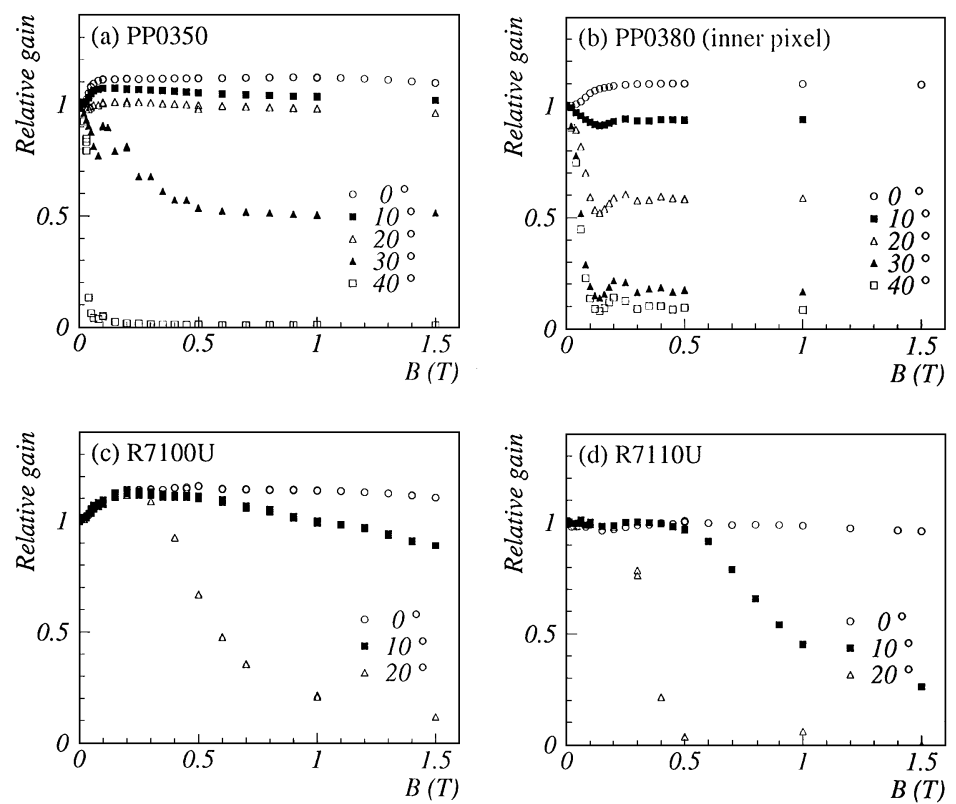

Fig. 11. Relative gains of (a) PP0350, (b) inner pixel of PP0380, (c) R7100U, and (d) R7110U in magnetic fields. The gains are normalized by those at $B=0 \mathrm{~T}$.

Table 3

HPD gains normalized by those without magnetic fields. The magnetic field strength was $1.0 \mathrm{~T}$ for PP0380 and $1.5 \mathrm{~T}$ for others. The spot size was $4 \mathrm{~mm} \phi$ for PP0350 and PP0380, and $1 \mathrm{~mm} \phi$ for R7100U and R7110U

\begin{tabular}{llllll}
\hline & $\theta=0^{\circ}$ & $\theta=10^{\circ}$ & $\theta=20^{\circ}$ & $\theta=30^{\circ}$ & $\theta=40^{\circ}$ \\
\hline PP0350 & 1.10 & 0.94 & 0.60 & 0.20 & 0.12 \\
PP0380 & 1.10 & 1.02 & 0.96 & 0.51 & 0.13 \\
PP0380 & 1.01 & 1.01 & 1.01 & 1.02 & 1.02 \\
R7100U & 1.11 & 0.87 & 0.12 & - & - \\
R7110U & 0.97 & 0.26 & 0.01 & - & - \\
\hline
\end{tabular}

${ }^{a}$ the response of the inner pixel.

${ }^{b}$ the responses summed over all pixels.

pass through the photocathode and hit the diode. We measured the gain of PP0350 operated in this mode for different magnetic field strengths and fixed tilt angle $\left(\theta=0^{\circ}\right)$. The result is shown in Fig. 13. It was confirmed that the diode was insensitive to the magnetic field. We also performed a similar measurement for APDs (Hamamatsu S5300 series). The gain variation is found to be less
Table 4

HPD gains at $\theta=0^{\circ}$ normalized by those without magnetic fields

\begin{tabular}{llllll}
\hline$B(\mathrm{~T})$ & 0.05 & 0.2 & 0.5 & 1.0 & 1.5 \\
\hline PP0350 & 1.08 & 1.11 & 1.12 & 1.12 & 1.10 \\
PP0380 & 1.01 & 1.10 & 1.10 & 1.10 & 1.10 \\
PP0380 & 1.01 & 1.01 & 1.01 & 1.01 & 1.01 \\
R7100U & 1.01 & 1.14 & 1.14 & 1.14 & 1.11 \\
R7110U & 0.98 & 0.97 & 0.99 & 0.99 & 0.99 \\
\hline
\end{tabular}

${ }^{a}$ the response of the inner pixel.

${ }^{b}$ the responses summed over all pixels.

than $1 \%$ with any $\theta$ and $B$, which is consistent with the result of HPDs in this mode.

In the phototube mode an HPD was operated as a phototube with no amplification. The photocathode voltage was applied, while the anode and the cathode of the diode were short-circuited. The photoelectrons were accelerated and collected to the diode. However, only the total charge of the photoelectrons was picked up as the signal regardless of their kinetic energies. The result for PP0350 




Fig. 12. Relation of the gain and the photocathode voltage in magnetic fields (PP0380). The gains are normalized by that of $V_{c}=10 \mathrm{kV}$ and $B=0 \mathrm{~T}$.

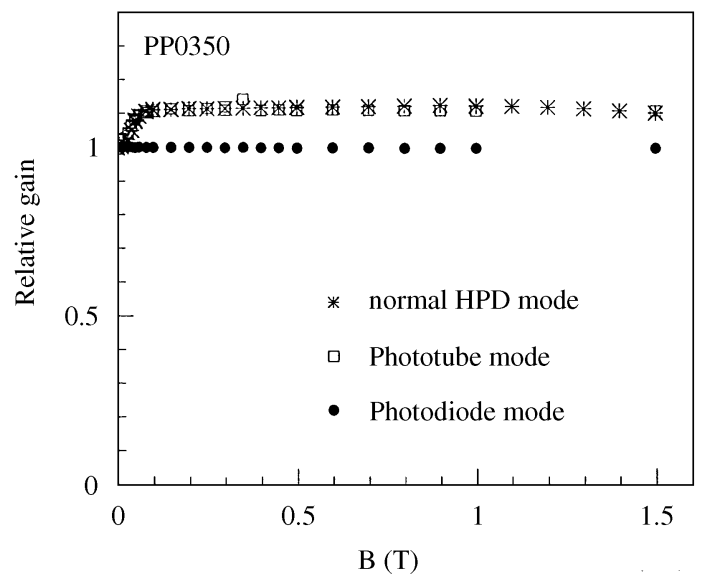

Fig. 13. Relative gains of PP0350 in magnetic fields $\left(\theta=0^{\circ}\right)$ operated in the photodiode mode, the phototube mode, and the normal HPD mode. The gains are normalized by those at $B=0 \mathrm{~T}$.

is also shown in Fig. 13. The behavior is very similar to that in the normal HPD operation mode.

\subsection{Origins of gain variation}

We confirmed that the performance of the diode did not change in magnetic fields. We also know that the quantum efficiency of the photocathode does not change in magnetic fields either [15].
Therefore, the gain variation of the HPD in magnetic fields should mainly originate from the movement of photoelectrons in the HPD.

As shown in Fig. 11a the gain of PP0350 increases to its maximum value at $B \sim 0.15 \mathrm{~T}$ for $\theta=0^{\circ}$. The gain is then kept nearly constant at least up to $B=1.5 \mathrm{~T}$. For non-zero $\theta$ values, gain reduction is observed at strong magnetic field and large $\theta$. Apart from the gain increase at $\theta=0^{\circ}$ the gain variation can roughly be explained by two effects $[3,4]$.

1. Shift of the photoelectron image on the diode. Part or all of the photoelectrons fail to bombard the effective area of the diode as a result of their cycloid motion in the electromagnetic field. This is the main origin of the gain decrease in magnetic fields.

2. Increase of the energy loss of the photoelectrons when they pass through the surface dead layer of the diode. The energy loss can be expressed as $\Delta E=\Delta E_{0} / \cos \eta$ where $\eta$ is the incident angle to the diode and $\Delta E_{0}$ is the energy loss of a photoelectron with $\eta=0^{\circ}$. This causes the steep dips of gain in low magnetic fields.

To explain the gain increase, an additional effect should be taken into account $[13,14]$.

3. Backscattering of photoelectrons at the surface of the diode. Part of the photoelectrons incident to the diode are backscattered. Without magnetic field, some scattered electrons escape from detection after a parabolic motion in the electric field. In contrast, in strong magnetic field, the scattered photoelectrons tend to return to the diode because they move along the magnetic field. This is the origin of the gain increase in magnetic fields.

The solid (dashed) curve in Fig. 14 is a result of calculation for $\theta=0^{\circ}\left(30^{\circ}\right)$ and $B=1.5 \mathrm{~T}$, where the three effects above are taken into account. In the simulation of backscattering of photoelectrons the model and data given in $[16,17]$ are used. The calculation reproduces the measurement well.

As for the Hamamatsu HPDs (see Fig. 11 c and d), the gain increase at $\theta=0^{\circ}$ was observed for R7100U, but not for R7110U. We consider that the disagreement between the measurement and expectation for R7110 is due to the aperture (focusing 




Fig. 14. Measured and simulated gain variations of PP0350. The soild (dashed) curve is a result of calculation for $\theta=0^{\circ}\left(30^{\circ}\right)$.

electrode) in front of the small-size APD. According to a calculation, possible imperfection in the setup such as a small tilt angle of the magnetic field, a small shift of the light spot position, and the finite light spot size, would result in the loss of electrons at the aperture structure. We assume that this is the reason of the small difference between the gains with and without magnetic fields at $\theta=0^{\circ}$.

Taking the three effects into account, all the measurements in magnetic fields can be consistently understood.

\section{Summary}

We tested four different types of the HPDs firstly without magnetic fields. We measured the absolute gains. The typical gain was 1500-3500 for normal HPDs, and 60000 for an avalanche-type HPD. The position uniformity was found to be excellent, although cross-talk signals were observed for a multi-pixel type. We confirmed that photoelectron counting was possible with the compact Hamamatsu HPDs.

Secondly we tested the four HPDs in magnetic fields. They were found to be operational at least up to $1.5 \mathrm{~T}$ with appropriate field directions. The behavior in magnetic fields is well understood by the three effects; cycloid motion, energy loss, and backscattering of photoelectrons.

\section{References}

[1] R. DeSalvo et al., Nucl. Instr. and Meth. A 315 (1992) 375.

[2] S. Basa et al., Nucl. Instr. and Meth. A 330 (1993) 93.

[3] H. Arnaudon et al., Nucl. Instr. and Meth. A 324 (1994) 558.

[4] G. Anzivino et al., Nucl. Instr. and Meth. A 365 (1995) 76.

[5] Y. Fujii et al., Nucl. Instr. and Meth. A 366 (1995) 71.

[6] E. Bosato et al., Nucl. Instr. and Meth. A 387 (1997) 97.

[7] C.P. Datema, Nucl. Instr. and Meth. A 387 (1997) 100.

[8] P. Cushman et al., Nucl. Instr. and Meth. A 387 (1997) 107.

[9] L. Waldron et al., Nucl. Instr. and Meth. A 387 (1997) 113.

[10] D. Websdale, Nucl. Instr. and Meth. A 387 (1997) 117.

[11] E. Chesi et al., Nucl. Instr. and Meth. A 387 (1997) 122.

[12] M. Suyama et al., IEEE Trans. Nucl. Sci. NS-44 (1997) 985.

[13] C. D'Ambrosio et al., Nucl. Instr. and Meth. A 338 (1994) 389.

[14] C. D’Ambrosio et al., Nucl. Instr. and Meth. A 345 (1994) 279.

[15] Hamamatsu Photonics K.K., private communications.

[16] G.D. Archard, J. Appl. Phys. 32 (1961) 1505.

[17] E.H. Darlington, J. Phys. D 8 (1975) 85. 